\title{
Properties of PVA/AA photopolymers at very low spatial frequencies
}

\author{
S. Gallego ${ }^{1}$, A. Márquez ${ }^{1}$, D. Méndez ${ }^{1}$, S. Marini ${ }^{1}$, E. Fernández ${ }^{2}$. \\ ${ }^{1}$ Dept. Física Enginyeria de Sistemes i Teoria del Senyal, Universitat d'Alacant (Spain) Apartat 99 \\ E-03080 Alacant
}

${ }^{2}$ Dept. Òptica, Farmacologia i Anatomia, Universitat d'Alacant (Spain) Apartat 99 E-03080 Alacant

\begin{abstract}
Polyvinyl/acrylamide (PVA/AA) photopolymers are often used as holographic recording materials. Depending on each particular application, different spatial frequencies can be recorded. The only limitation appears when high spatial frequencies are stored (over 2500 lines $/ \mathrm{mm}$ the diffraction efficiencies achieved are very low). This cut-off spatial frequency is due to the finite size of polymer chains. On the other hand, in the case of very low spatial frequencies, monomer diffusion times are highly increased and so there are important distortions in the shape of the gratings stored. In order to characterize the hologram formation in these types of materials, many parameters must be taken into account. One of the most important parameters to measure is the diffusion of the molecules inside the layer. In this sense, when very low spatial frequencies are recorded, component diffusion and thickness variations can be determined more accurately. In previous works, we have characterized the photopolymer at the zero spatial frequency limit, where diffusion does not take place, measuring the refractive index variations and the shrinkage. In this work we have recorded different long period gratings (at very low spatial frequencies diffusion starts to be important) and we have analyzed the profiles formed in the material during exposure and the refractive index distributions. This study has been carried out measuring the transmission and reflection orders of diffractive gratings. Furthermore, to obtain a deeper insight into the profile of the gratings recorded and especially into the sharpness of their edges, we have obtained numerically the diffraction-efficiency values fitting a Fermi-Dirac function to each profile.

Keywords: Recording materials, photopolymers, diffractive gratings
\end{abstract}

\section{INTRODUCTION}

Photopolymers have many different applications in optics due to their good properties as optical recording medium. Large range of spatial frequencies can be recorded in photopolymer systems, from zero spatial frequency to more than 4000 lines/mm [1]. In particular the chemical photopolymer composition is modified for each particular application [2]. For example, to design holographic high spatial frequencies are required; to achieve this goal short polymer chains are needed. On the other hand long polymer chains are interesting to achieve high diffraction efficiencies at low spatial frequencies, due to the high values of refractive index produced when long polymer chains are created.

In the last ten years, some studies have been carried out dealing with the recording of very low spatial frequencies (less than 10 lines/mm) in PVA/AA based layers[3-7]. In Ref. [6] a contact-copying process was used to transfer low spatial frequency diffractive optical elements (DOEs) from binary amplitude masks[7] onto the photopolymer, with good results. The same technique was used to analyze the suitability of a wide range of holographic recording materials at low spatial frequencies so as to register DOEs and optical correlation filters [8]. In this work we proposed a hybrid opticaldigital setup incorporating a liquid crystal display (LCD) to generate phase DOEs onto photopolymers. The application of photopolymers to DOEs generation requires the characterization of the modulation properties of the material realised in previous works.

To record the diffractive elements onto the material it becomes practical to use a spatial light modulator [5]. The Liquid Crystal Display (LCD) used permits us to modify the properties (phase and intensity) of the incident wave front [9]. In particular the intensity of the transmittance modulation is used for the present studies. We can record different diffractive optics elements systematically. In this work we focus our attention in the material behaviour when different spatial frequencies are recorded. We analyze the changes in the shape of the phase gratings obtained during the recording process. To obtain a deeper insight into the profile of the generated gratings and especially into the sharpness of the edges and the phase modulation depth, we have obtained numerically the diffraction-efficiency values for the different orders using a Fermi-Dirac function based profile [6]. Fitting the numerical results with the experimental ones we can sketch the grating profile. Besides, we can also evaluate the importance of the species diffusion for the shape of the 
edges. In particular we study the material behavior for the following spatial grating periods: $0.168 \mathrm{~mm}, 0.336 \mathrm{~mm}$ and $0.672 \mathrm{~mm}$ (i.e. the spatial frequencies are between less than 2 lines $/ \mathrm{mm}$ to 6 lines $/ \mathrm{mm}$ ). These long periods are in the range of the diffusion length for the monomer.

\subsection{Experimental Set-up}

\section{EXPERIMENTAL}

The experimental set-up used is shown in figure 1. We use an expanded, collimated recording beam provided by a solidstate Verdi laser (Nd-YVO4) with a wavelength of $532 \mathrm{~nm}$ (at this wavelength the dye exhibits maximum absorption). The exposure intensity is $0.5 \mathrm{~mW} / \mathrm{cm} 2$. The periodic pattern is introduced by a liquid crystal display (LCD), a Sony LCD model LCX012BL, extracted from a video projector Sony VPL-V500. We use the electronics of the video projector to send the voltage to the pixels of the LCD. The LCD is used in the amplitude-mostly modulation regime by proper orientation of the external polarizers $(\mathrm{P})[10]$; then the pattern is imaged onto the material with an increased spatial frequency (a demagnifying factor of 2). The use of the LCD allows us to change the period of the grating recorded in the photopolymer without moving any mechanical part of the set-up. To analyze in real time the variation in efficiency of the different diffraction orders, we use an unexpanded beam of a He-Ne laser $(633 \mathrm{~nm})$ incising at a small angle with respect to the normal axis of the recording material. A diaphragm (stop2) is placed in the focal plane of the relay lens so as to eliminate the diffraction orders produced by the pixelation of the LCD. In this work the patterns sent to the LCD correspond to binary gratings with different periods. It can be expected that the final pattern imaged onto the recording material will be partially low filtered due to the finite aperture of the imaging system and especially due to the filtering produced by stop2. However the periods of the gratings addressed onto the LCD have been kept long enough so that we can still consider that the image on the recording plane is rather close to a binary grating. In the results in section 3.3 it will become clear that at least for low exposition times in the recording process this binary profile is basically maintained when registered into a phase profile.

To obtain the Fourier transform of the pattern, to measure the diffracted intensities, we place a lens after the grating (recorded in the photopolymer). The Fourier plane is captured using a CCD camera at the focal plane of the lens. We use the CCD camera model pco.1600 from pco.imaging. This is a high dynamic14 bits cooled CCD camera system with a resolution of 1600x1200 pixels, and a pixel size of 7.4x7.4 $\mathrm{m} 2$. The high dynamic range together with the high number of pixels allows using this camera as a radiometer where the intensity directed to the various diffraction orders (in our case 9 orders) can be measured in parallel. We have experimentally estimated the error of the diffraction efficiencies obtained using the camera and it turns out to be around $0.5 \%$.

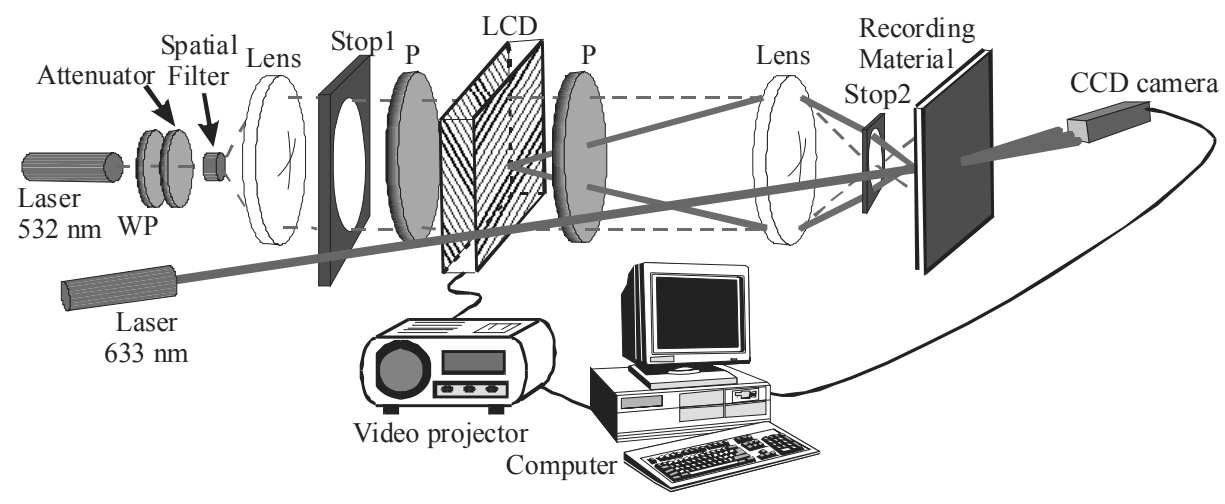

Fig. 1. Experimental set-up (transmission measurements). The recording material is exposed to the green laser beam $(\lambda=532 \mathrm{~nm})$ and zero order diffraction efficiency is measured with the red beam $(\lambda=633 \mathrm{~nm})$. P correspond to the polarizers and WP to the wave plate. 
The photopolymer fabricated to store the gratings is similar to the ones used in previous works [4], in this paper we only analyze the composition without crosslinker. The PVA/AA formulations contain a dye (yellowish eosin), a cosensitizer which is triethanolamine (TEA), one monomer AA and a polymer as a binder (PVA). The thickness of the layers studied in this work is $100 \pm 5 \mu \mathrm{m}$

\subsection{Fitted Profile}

The Fermi-Dirac based profile is a better option than the super-Gaussian based profile used in previous works [6], for two many reasons. The parameters in the Fermi-Dirac function are continuous whereas in the super-Gaussian function the parameter describing the sharpness of the edges is discrete, thus there is no gradual transition between the fitted profiles. Furthermore, the Fermi-Dirac function provides a very good fit to the cosine function, which may be considered as a reasonable limiting case when edges become highly smoothed by diffusion. The mathematical expression for the amplitude transmission of a transparent phase element showing a Fermi-Dirac phase profile can be written as follows,

$$
\begin{gathered}
t(x)=\exp (i \varphi(x)) \\
\varphi(x)=\varphi_{0}\left(1+\exp \left(\alpha \cdot\left(\frac{|x|}{\Omega}-1\right)\right)\right)^{-1}
\end{gathered}
$$

where 0 is the asymptotic phase modulation depth (for an $\mathrm{x}$-interval $(-\infty,+\infty)$ ), $\Omega$ controls the symmetry (or duty cycle) of the profile, and $\alpha$ is the parameter characterizing the function shape and sharpness of the edges. The function is limited to an $\mathrm{x}$-interval $(-1,1)$, and repeated periodically. We consider symmetric gratings, which for this $\mathrm{x}$-interval correspond to the value $\Omega=0.5$. We rescale the phase values obtained in the $\mathrm{x}$-interval $(-1,1)$ so that the phase modulation depth in this finite interval equals the asymptotic phase modulation depth. In figure 2(a) we show the normalized profile for the Fermi-Dirac function in the x-interval $(1,-1)$, for $\Omega=0.5$ (symmetric profile), and for different values of $\tilde{\alpha}$ We see that for $\alpha=1$ the profile corresponds to a triangle function, for $\alpha=3$.3 is close to a cosine function and with the increase in $\alpha$ the profile approaches a rectangle function. In figure 2(b) we compare the profile given by a super-Gaussian and a Fermi-Dirac function to a cosine function. We see that the Fermi-Dirac function with $\alpha=3.3$ is a good approximation to the cosine. The best super-Gaussian fit is given by the Gaussian function itself [6] which is clearly a worse approximation.
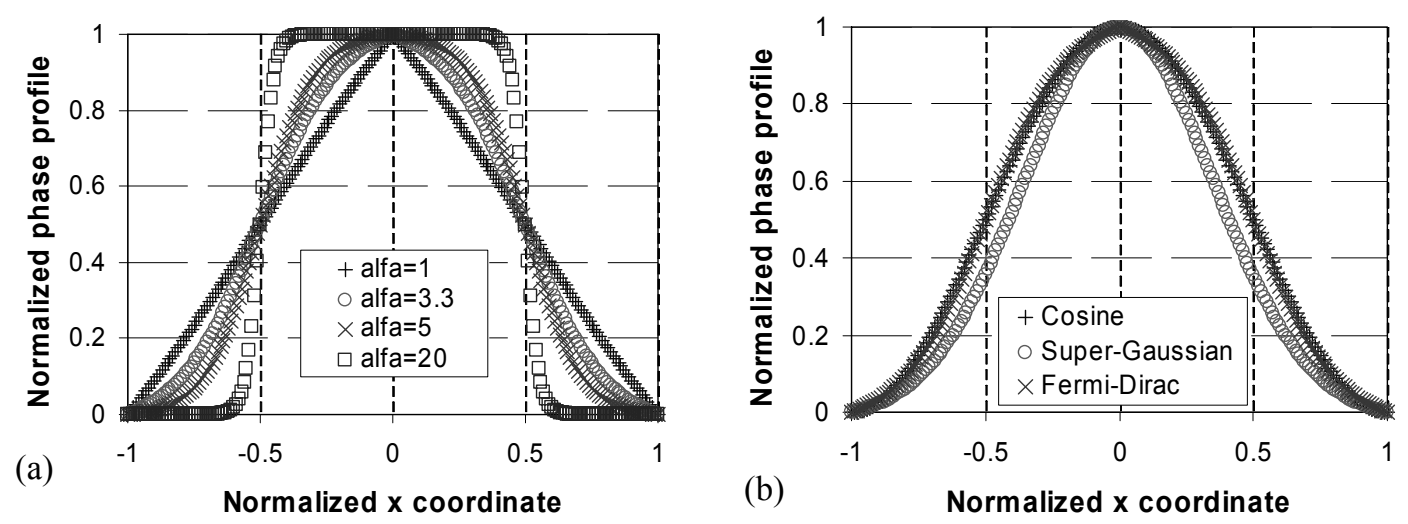

Figure. 2. Normalized phase profiles. (a) Fermi-Dirac profiles for different values of $\alpha$ and for $\Omega=0.5$ (symmetric gratings). (b) Super-Gaussian and Fermi-Dirac best fits to a cosine function, where we show that the Fermi-Dirac function (with $\alpha=3.3$ ) provides a very good approximation.

We have compared the diffraction efficiencies calculated numerically using the Fermi-Dirac model with the experimental values for the first 9 orders (orders $0,1, \ldots$, and 8). In next section we will show these results that have 
allowed obtaining the profile, both in transmission and in reflection, for the phase gratings stored in the photopolymer material.

\section{RESULTS AND DISCUSSION}

As we already remarked in the introduction, phase grating can be stored in photopolymers due two phenomena: the volume changes in the surface and the refractive index variation in the polymerization zones. In this section we analyze the two effects together studying the transmitted orders.

Recent works based on interferometric optical surface profilometers $[3,11]$ have demonstrated the existence of a thickness increase in the illuminated areas of some photopolymers for spatial frequencies of 10 lines per mm. This swelling is due to the incorporation of monomer molecules from the dark zones by diffusion processes. On the other hand shrinkage of around $4 \%$ has been measured at the zero spatial frequency limit [5] where diffusion does not take place. For spatial periods longer than 0.672 we can expect a similar behaviour to the one observed at the zero spatial frequency limit.

In figure 3 we present the diffractive efficiency for the first four orders for different grating periods $(0.168,0.336$ and $0.672 \mathrm{~mm}$ ) as a function of the exposure time. The most significant fact sketched in the figure is the instability of the diffraction efficiencies after long exposure times. For the $0.16 \mathrm{~mm}$ case, the stabilization of diffraction efficiencies cannot be observed after $500 \mathrm{~s}$ (corresponding to about $250 \mathrm{~mJ} / \mathrm{cm}^{2}$ ). This fact constitutes an important difference in comparison with the zero frequency limit case [20], where after $200 \mathrm{~s}$ (about $80 \mathrm{~mJ} / \mathrm{cm}^{2}$ ) the phase shift becomes constant. The phenomenon can be explained taking into account the influence of the species diffusion in the refractive index distribution and in the layer profile [5]. In particular there exists a mixture of three different effects: the changes in the refractive index, the shrinkage and the swelling of the material. The changes in the refractive index of the material are due to polymerization and the existence of holes [12]. Shrinkage is due to the smaller volume of poly-acrylamide chains compared to the volume of the same amount of monomer distributed individually. Swelling can be related to species diffusion from the dark layer areas to the bright ones. The swelling is maximum for periods around $0.1 \mathrm{~mm}$ [4], although it depends on the periods of the gratings recorded [4]. We observe a smaller material sensitivity (defined as the necessary energy to achieve the first minimum of diffraction efficiency of the zero order) for long periods $(0.672 \mathrm{~mm})$, fact that indicates a minor change in the material surface due to the compensation between diffusion and shrinkage. On the last place, it is important to note the high values of diffraction efficiencies achieved for thin gratings, larger than $20 \%$ in the first two cases. This parameter is fundamental to use the material to record DOEs. In principle, generic DOEs may be thought as a composition of different spatial frequencies. In this sense it would be desirable to obtain similar diffraction efficiencies and sensitivities for the various frequencies. From the results in figure 3 we see that the values of these parameters are different for the various frequencies. In this case, a trade-off value for the time exposure providing a high diffraction efficiency across the spatial frequency range of interest should be considered when recording a generic DOE. 

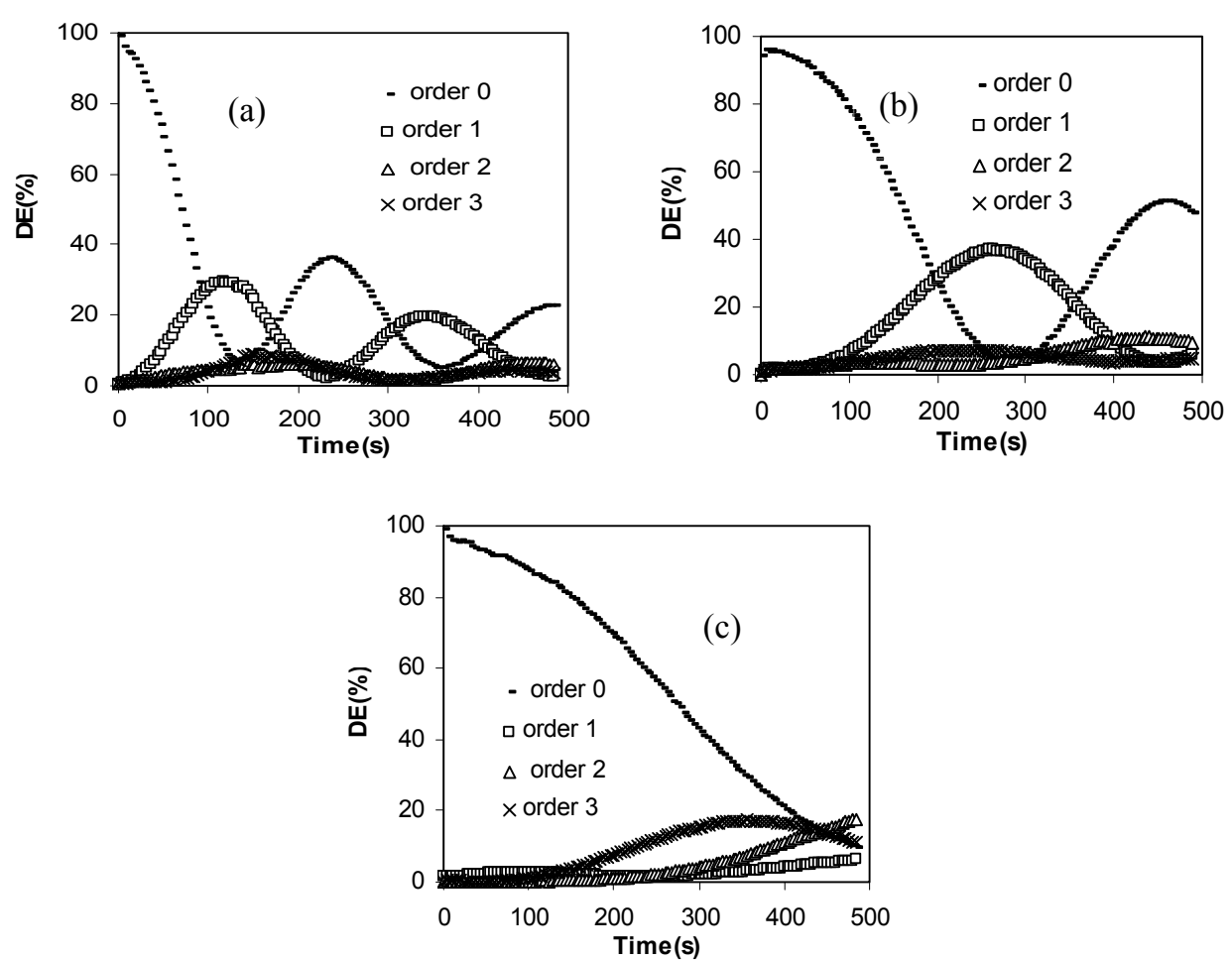

Figure 3.- Diffraction efficiencies of the first four orders for different gratings periods $(0.168,0.336$ and $0.672 \mathrm{~mm}$, figures a, $\mathrm{b}$ and $\mathrm{c}$ respectively) as a function of the exposition time.

To study the phase gratings, we have used a simulated profile based on the Fermi-Dirac function. Usually, in the initials steps of recording $(10 \mathrm{~s})$ only $-1,0$ and +1 diffraction orders can be detected, what implies that we cannot analyze the shape of the profile without making more assumptions. In this sense, there are two important variables that we must take into account in order to characterize the phase gratings profiles recorded in the photopolymers: the depth of the phase shift and the shape of the edges of the grating stored. After a few seconds more pairs of orders become significant and so we can fit the experimental data and obtain a more reliable profile of the phase modulation. Specifically the used procedure considers nine diffracted orders, from order 0 to 8 . In figure 4 we present the profile evolution during exposure for lowest and highest spatial periods. As we can see the smoothing of the edges begins firstly for low spatial periods, due to the higher influence of molecules diffusion inside the material. 

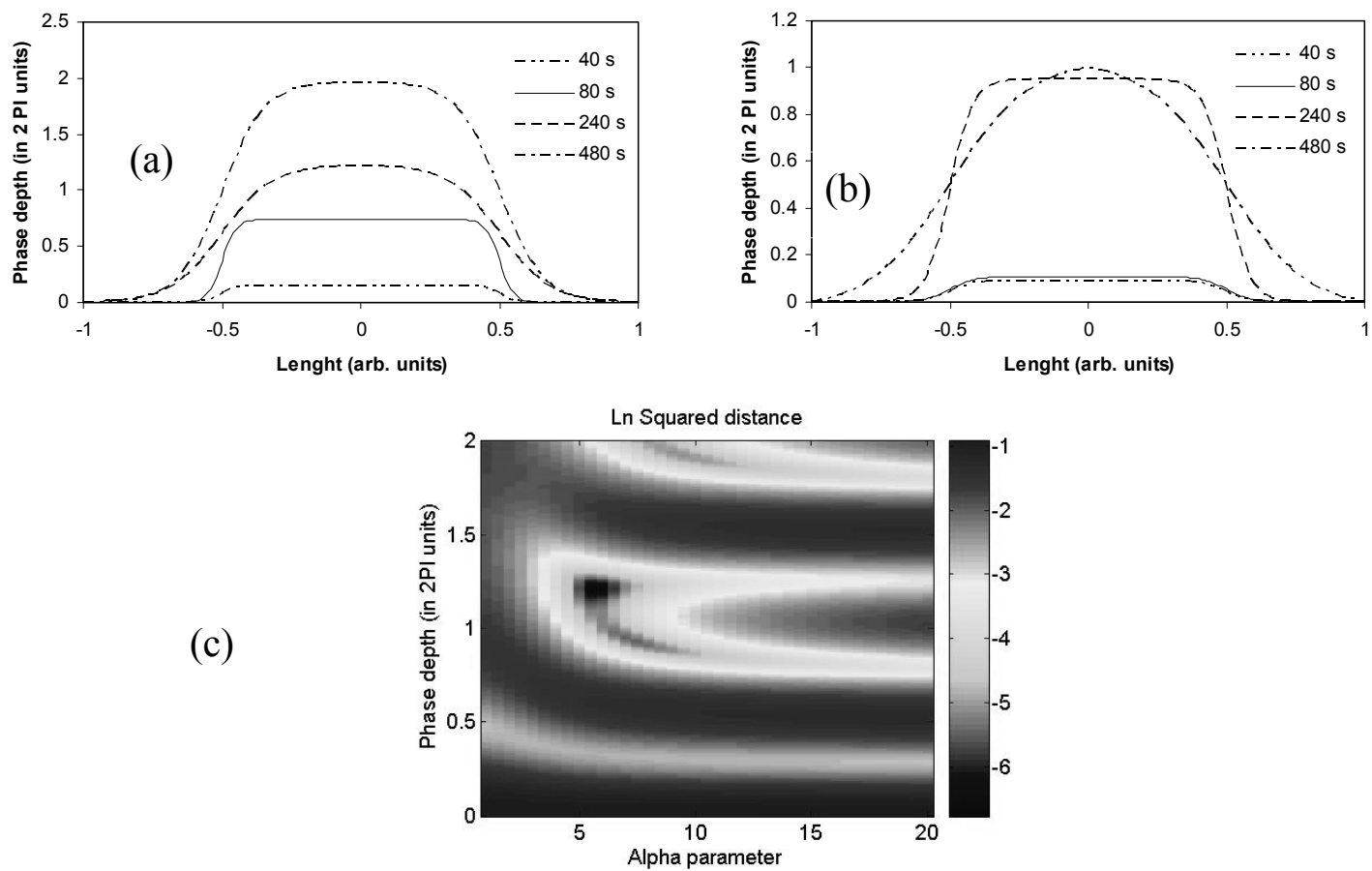

Figure 5.- Fitted surface profiles for different exposure times using Fermi-Dirac distribution (105 $\mu \mathrm{m}$ thick, in transmission). a) Spatial period of $0.168 \mathrm{~mm}$. b) Spatial period of $0.672 \mathrm{~mm}, \mathrm{c}$ ) Search for the optimum phase profile after $240 \mathrm{~s}$.

As we have mentioned in the introduction, when photopolymer is illuminated, there are refractive index changes and also material volumes variations. To have an approximate idea of these two effects we have analyzed the efficiency of the reflected diffracted orders which will specifically show material volume variations. The diffraction efficiencies of the first four diffracted orders as a function of the exposure time are depicted in figure 5 for the same three spatial frequencies. We observe a similar behaviour for the gratings with periods of 0.336 and $0.672 \mathrm{~mm}$ due to the almost null influence of molecules diffusion for these long periods. On the other hand, we observe clear differences in the first $200 \mathrm{~s}$ for the grating with a period of $0.168 \mathrm{~mm}$. In the reflection case high values of diffraction efficiency of the first diffracted order (higher of $20 \%$ in all the cases) are achieved for these thin layers. 

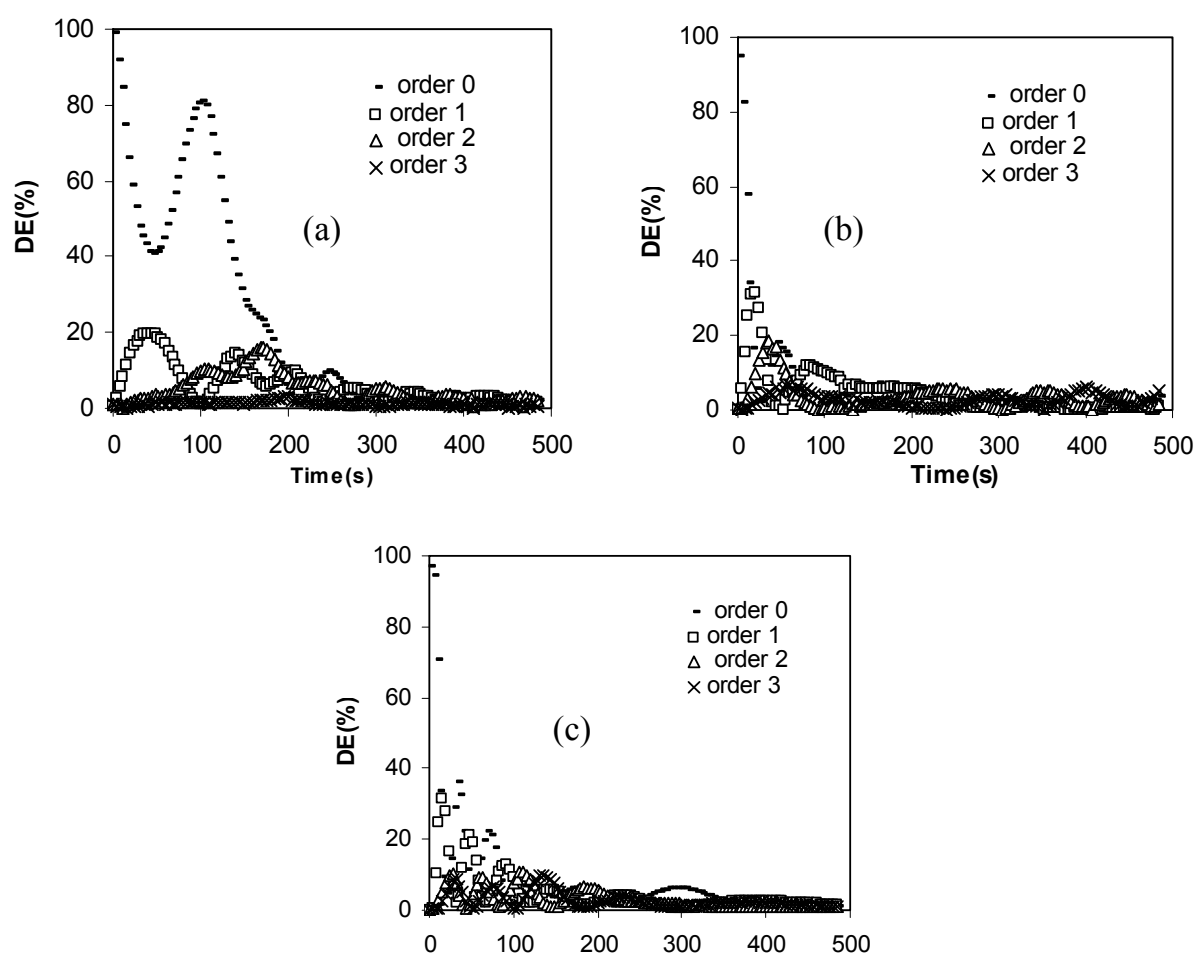

Figure 6.- Diffraction efficiencies of the first four reflected orders for different gratings periods $(0.168,0.336$ and $0.672 \mathrm{~mm}$, figures a, $\mathrm{b}$, and c respectively) as a function of the exposition time.

At this point, it is important to remark that for all different spatial frequencies, the efficiencies of each order decrease to values lower than a $10 \%$ after $200 \mathrm{~s}$; this fact did not occur in the case of the transmitted orders as we have already observed in the previous section. Experimentally we obtain that as the time increases the total sum of the intensity diffracted to the first 17 orders $(0, \pm 1, \ldots, \pm 8)$ becomes close to zero. This is an unexpected result since intuitively it seems that a significant portion of the energy should be contained in the first diffracted orders. To gain some insight into the origin of these results we have used the Fermi-Dirac model presented in Section 2.2 to calculate the sum of the intensity directed to the first 17 orders as a function of the phase depth of the grating and for different values of the $\alpha$ parameter. In figure 6 we have represented these results. As can be seen for values of $\alpha$ lower than 5 the first 8 diffraction orders are very weak when the phase depth is large. Therefore, this effect indicates that large values of the phase modulation are obtained and that they correspond to smoothed profiles (see figure 2(a) for the profile corresponding to $\alpha=5$ ). In reference [5] we demonstrated experimentally than the phase depth can be higher than $10 \pi$ radians. In general, thin gratings with such large modulation depths are not produced, since typically the range of interest is about $2 \pi$ radians, i.e. very far from the more than $10 \pi$ radians achievable in reflection with the photopolymer under analysis. To our knowledge this theoretical and experimental analysis for thin phase gratings with very large phase modulations has not been done till now. 


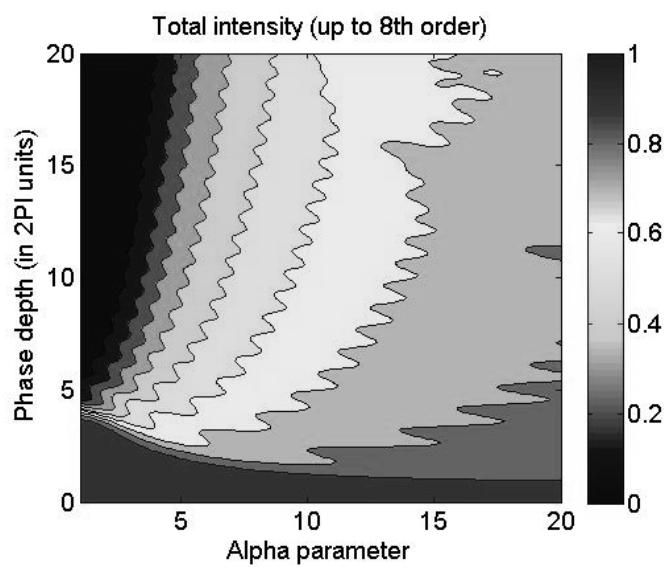

Figure 7.- Sum of diffraction efficiencies of the first 8 reflected orders for different values of $\alpha$ and phase depth.

\section{CONCLUSIONS}

In this work we have tested the feasibility of a PVA/AA based photopolymer to record structures with long periods. In particular, we have studied the critical range of spatial frequencies where species diffusion starts to play an important role in the phase image formation. We have analyzed the different behaviour observed at three different spatial frequencies $(5.8,2.9$ and 1.49 lines $/ \mathrm{mm})$. Using a Fermi-Dirac function; we have reported the shape of the profiles and have estimated the phase depth. In this sense, we have shown that, due to diffusion processes, it is not possible to obtain sharp edges with PVA/AA based materials as exposition time increases. Techniques to fix the phase images, to decrease the diffusion, are required to stop the smoothing of the edges. Furthermore, we have demonstrated that the low values of diffracted intensity obtained for the first nine orders are due to the combination of a very large phase depth and a smoothed profile.

\section{ACKNOWLEDGEMENTS}

This work was supported by the "Ministerio de Edicación y Ciencia" (Spain) under projects FIS2008-05856-C02-01 and FIS2008-05856-C02-02 and by Generalitat Valenciana under project GVPRE/2008/137

\section{REFERENCES}

[1] R. Jallapuram, I. Naydenova, S. Martin, R. Howard, V. Toal, Sven Frohmann, Susanna Orlic, Hans J Eichler, "Acrylamide based photopolymer for micro-holographic data storage", Optical Materials, 28 , 1329-1333, (2006).

[2] Michael R. Gleeson, Dusan Sabol, Shui Liu, Ciara E. Close, John V. Kelly, and John T. Sheridan, "Improvement of the spatial frequency response of photopolymer materials by modifying polymer chain length," J. Opt. Soc. Am. B 25, 396-406 (2008)

[3] I. Naydenova, E. Mihaylova, S. Martin, and V. Toal, "Holographic patterning of acrylamide-based photopolymer surface," Opt. Express 13, 4878-4889 (2005)

[4] S. Gallego, A. Márquez, D. Méndez, M. Ortuño, C. Neipp, M. L. Alvarez, A. Beléndez, E. Fernández and I. Pascual, "Real-time interferometric characterization of a PVA based photopolymer at the zero spatial frequency limit” Appl. Opt. 46, 7506-7512 (2007).

[5] S. Gallego, A. Márquez, D. Méndez, M. Ortuño, C. Neipp, M. L. Alvarez, A. Beléndez, E. Fernández and I. Pascual, "Analysis of PVA/AA based photopolymers at the zero spatial frequency limit using interferometric methods" Appl. Opt. 47, 2556-2563 (2008)

[6] I. Pascual, A. Márquez, A. Beléndez, A. Fimia, J. Campos and M. J. Yzuel, "Copying low spatial frequency diffraction gratings in photopolymer as phase holograms", J. Mod. Opt. 47, 1089-1097 (2000).

[7] A. Márquez, J. Campos, M. J. Yzuel, I. Pascual, A. Fimia and A. Beléndez, "Production of computer-generated phase holograms using graphic devices: application to correlation filters", Opt. Eng. 39, 1612-1619 (2000). 
[8] A. Márquez, C. Neipp, A. Beléndez, J. Campos, I. Pascual, M. J. Yzuel and A. Fimia, "Low spatial frequency characterization of holographic recording materials applied to correlation", J. Opt. A: Pure Appl. Opt. 5, S175S182 (2003).

[9] A. Márquez, S. Gallego, D. Méndez, M. L. Álvarez, E. Fernández, M. Ortuño, C. Neipp, A. Beléndez, I. Pascual, "Application of a liquid crystal display to generate diffractive optical elements onto a photopolymer", Proc. EOS Topical Meeting on Diffractive Optics, 194-195 (2007).

[10] A. Márquez, C. Iemmi, I. Moreno, J. A. Davis, J. Campos and M. J. Yzuel, "Quantitative prediction of the modulation behavior of twisted nematic liquid crystal displays," Opt. Eng. 40, 2558-2564 (2001).

[11] T. Babeva, I. Naydenova, S. Martin, and V. Toal, "Method for characterization of diffusion properties of photopolymerisable systems" Optics Express 16, 8487-8497 (2008).

[12] John Kelly, Michael Gleeson, Ciara Close, Feidhlim O'Neill, John Sheridan, Sergi Gallego, and Cristian Neipp, "Temporal analysis of grating formation in photopolymer using the nonlocal polymerization-driven diffusion model," Opt. Express 13, 6990-7004 (2005) 